\title{
Hybrid Beamforming for Terahertz Joint Ultra-Massive MIMO Radar-Communications
}

This paper was downloaded from TechRxiv (https://www.techrxiv.org).

\section{LICENSE}

$\mathrm{CCO}$

SUBMISSION DATE / POSTED DATE

$27-12-2021 / 29-12-2021$

\section{CITATION}

Elbir, Ahmet M.; Mishra, Kumar Vijay; Chatzinotas, Symeon (2021): Hybrid Beamforming for Terahertz Joint Ultra-Massive MIMO Radar-Communications. TechRxiv. Preprint.

https://doi.org/10.36227/techrxiv.17694467.v1

$\mathrm{DOI}$

10.36227/techrxiv.17694467.v1 


\title{
Hybrid Beamforming for Terahertz Joint Ultra-Massive MIMO Radar-Communications
}

\author{
Ahmet M. Elbir ${ }^{1}$, Kumar Vijay Mishra ${ }^{2}$ and Symeon Chatzinotas ${ }^{1}$ \\ ${ }^{1}$ University of Luxembourg, Luxembourg \\ ${ }^{2}$ United States CCDC Army Research Laboratory, Adelphi, MD 20783 USA \\ E-mail: ahmetmelbir@gmail.com, kumarvijay-mishra@uiowa.edu, symeon.chatzinotas@uni.lu
}

\begin{abstract}
In this paper, we investigate the hybrid beamforming problem in joint radar-communications at terahertz $(\mathrm{THz})$ bands. In order to address the extremely high attenuation at THz, ultra-massive multiple-input multiple-output (UM-MIMO) antenna systems have been proposed for $\mathrm{THz}$ communications to compensate propagation losses. Further, we propose a new group-of-subarrays (GoSA) UM-MIMO structure to reduce the hardware cost. We formulate the GoSA beamformer design as an optimization problem to provide a trade-off between the unconstrained communications beamformers and the desired radar beamformers. Numerical experiments demonstrate that the proposed approach outperforms the conventional approaches in terms of spectral efficiency and hardware costs.
\end{abstract}

Index Terms-Joint radar-communications, terahertz, hybrid beamforming, ultramassive MIMO.

\section{INTRODUCTION}

The millimeter-wave (mmWave) spectrum has been extensively studied to address the demands for high data rates in the fifth-generation $(5 \mathrm{G})$ wireless communications $[1,2]$. The maximum mmWave bandwidth being tens of $\mathrm{GHz}$, it is not possible to achieve data rates of the order of terabitsper-second $(\mathrm{Tb} / \mathrm{s})$ without significantly enhancing the current physical-layer efficiency. As a result, the future sixthgeneration $(6 \mathrm{G})$ networks are expected to exploit the $\mathrm{THz}$ frequencies $(0.3-10 \mathrm{THz})$ [3], where hundreds of $\mathrm{GHz}$ bandwidth is available to facilitate $\mathrm{Tb} / \mathrm{s}$ rates without dramatic efficiency increase in the physical-layer. There is, therefore, considerable interest in developing $\mathrm{THz}$ wireless solutions.

Higher bandwidths also result in improved radar range resolution. At present, mmWave radars with a few $\mathrm{GHz}$ bandwidths such as those used in automotive applications at 24 and $77 \mathrm{GHz}$ are unable to yield high-resolution images compared to the optical sensors. Higher operating frequency have smaller antenna apertures and microwave components, which is beneficial for radar deployment on cost and areasensitive platforms such as drones and ground vehicles. At $\mathrm{THz}$, the physical aperture of the antenna is expected to be very small and the availability of large transmit bandwidth has the potential to offer image resolutions closer to that from the optical sensors.

For $\mathrm{THz}$ communications, high propagation losses and power limitations are compensated by the beamforming gains obtained through deployment of extremely dense nano-antenna arrays [4], which may be based on graphene plasmonics [5] or metasurfaces [6]. Analogous to the developments in mmWave communications, [7] proposed a $\mathrm{THz}$ ultramassive multipleinput multiple-output (UM-MIMO) architecture that employs an array-of-subarrays (AoSA) of nano-transceivers to increase the coverage and improve the data rates. Various follow-up works (see, e.g., [4] for an overview) showed further UMMIMO enhancements through waveform design, beamforming, and resource allocation.

With this recent rise of both radar and communications applications at $\mathrm{THz}$, it has been suggested [8] to integrate radar sensing and communications functionalities in future wireless $\mathrm{THz}$ systems to facilitate spectrum sharing, enhance pencil beamforming, save hardware cost, and improve resource usage. This follows recent efforts in realizing such joint radar-communications (JRC) architectures at mmWave [2], including for ultrashort ranges, joint MIMO-radar-MIMOcommunications, and distributed MIMO JRC [9]. In this paper, we focus on a UM-MIMO structure for JRC at $\mathrm{THz}$ band.

Analogous to their massive MIMO counterparts at mmWave, the UM-MIMO architecture implies that fully digital beamforming is infeasible because of huge associated cost, area, and power. This necessitates use of hybrid beamforming [10], wherein the signal is processed by both analog and digital beamformer. Although some recent works [11] investigate $\mathrm{THz}$ hybrid beamformer designs, they do not examine it in the context of the recently proposed, practically feasible UMMIMO, and more so, its application in THz JRC.

To reduce the hardware complexity, we propose a group of subarrays (GoSAs) structure, in which the antenna elements in the same subarray are connected to the same phase-shifter. Thus, the proposed structure employs even fewer phaseshifters than that of fully-connected (FC) arrays or partiallyconnected (PC) AoSA structures [12, 13], while providing satisfactory radar and communications performance in terms of the beampattern and the spectral efficiency, respectively. In order to improve the radar performance, the higher degrees of freedom (DoF) are provided by using PC GoSAs. Nevertheless, $\mathrm{PC}$ structure has poor spectral efficiency performance compared to the FC array. Hence, we suggest a PC with overlapped (PCO) GoSA structure for performance improvement. To design the hybrid beamformers based on the PCO structure, we propose a modified version of the manifold optimization (MO)-based alternating minimization (AltMin) technique [14], which is originally suggested to solve the beamformer design problem in FC arrays. Our numerical experiments show that 


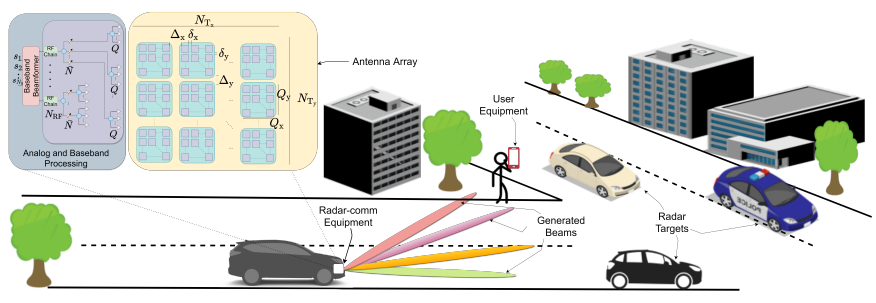

Fig. 1. A radar-communications system for a vehicle-to-vehicle (V2V) and vehicle-to-device (V2D) scenario, wherein a single $\mathrm{THz}$ radarcommunications unit, with a $N_{\mathrm{T}}=N_{\mathrm{T}_{\mathrm{x}}} \times N_{\mathrm{T}_{\mathrm{y}}}$ antenna array, is mounted onto a vehicle to simultaneously transmit toward both communications receiver and vehicular targets.

the proposed approach has much lower hardware complexity than the state-of-the-art techniques, while maintaining satisfactory radar and communications performance.

\section{System Model And PRoblem Formulation}

We consider a UM-MIMO architecture with a JRC system for a vehicle to vehicle (V2V) and vehicle to device (V2D) scenario, in which the transmitter (TX) senses the environment via probing waveforms to the targets and communicates with the receiver (RX), as illustrated in Fig. 1. The antenna arrays at the TX and the RX employ graphene-based plasmonic nanoantennas, which are placed on a metallic surface layer, with a dielectric layer between them [7, 12]. The antennas form a groups of subarrays (GoSAs) structure as each subarray consists of $Q_{\mathrm{x}} \times Q_{\mathrm{y}}$ uniform rectangular arrays (URAs) with $Q=Q_{\mathrm{x}} Q_{\mathrm{y}}$ antennas, as shown in Fig. 1. Also, there are $N_{\mathrm{T}}=N_{\mathrm{T}_{\mathrm{x}}} N_{\mathrm{T}_{\mathrm{y}}}$ and $N_{\mathrm{R}}=N_{\mathrm{R}_{\mathrm{x}}} N_{\mathrm{R}_{\mathrm{y}}}$ subarrays of size $Q$ at the TX and RX, respectively, which form an $N_{\mathrm{T}} Q \times N_{\mathrm{R}} Q$ UM-MIMO transceiver architecture. In each $Q_{\mathrm{x}} \times Q_{\mathrm{y}}$ subarray, the antenna spacing along the $x$ - and $y$-axis are $\delta_{\mathrm{x}}, \delta_{\mathrm{y}}$ and the distance between each subarray are $\Delta_{\mathrm{x}}, \Delta_{\mathrm{y}}$, respectively.

In the downlink, the TX with $N_{\mathrm{T}}$ subarrays, each of which has $Q$ antenna elements, aims to transmit $N_{\mathrm{S}}$ data streams towards the RX in the form of $\mathbf{s}=\left[s_{1}, \ldots, s_{N_{\mathrm{S}}}\right]^{\top}$ by using hybrid analog digital beamformers with $N_{\mathrm{RF}} \mathrm{RF}$ chains, where $\mathbb{E}\left\{\mathbf{s s}^{\mathrm{H}}\right\}=\mathbf{I}_{N_{\mathrm{S}}}$ and $N_{\mathrm{S}} \leq N_{\mathrm{RF}}$. Due to beamforming at subarray level, each subarray of size $Q$ generates a single beam [7]. This is done by connecting the $Q$ antennas in each subarray to a single phase-shifter to lower the hardware complexity. Thus, the TX first applies an $N_{\mathrm{RF}} \times N_{\mathrm{S}}$ baseband precoder $\mathbf{F}_{\mathrm{BB}}$. Then, the signal is passed through an $\mathrm{RF}$ precoder $\mathbf{F}_{\mathrm{RF}} \in \mathbb{C}^{N_{\mathrm{T}} \times N_{\mathrm{RF}}}$ by employing $N_{\mathrm{T}}$ phase-shifters, as shown in Fig. 2c. In conventional FC structure (see Fig. 2a), each antenna is connected to $N_{\mathrm{RF}} \mathrm{RF}$ chains while the AoSA model in Fig. 2b has PC structure and it connects each RF chain to $\bar{N} Q$ antennas in each subarray, where $\bar{N}=\frac{N_{\mathrm{T}}}{N_{\mathrm{RF}}}[12]$. In this work, we propose a GoSA architecture, as shown in Fig. 2c, in which $N_{\mathrm{T}} Q$ antennas are partitioned into $N_{\mathrm{RF}}$ groups, each of which has $\bar{N} Q$ antennas. Also, each group consists of $\bar{N}$ subarrays of size $Q$. We assume that the antennas in each subarray are fed with the same phase shift to reduce

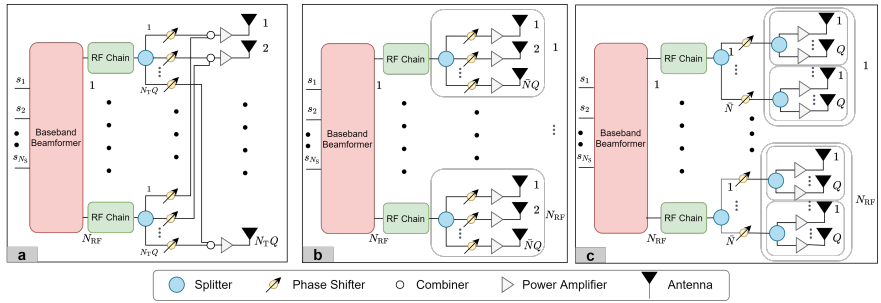

Fig. 2. Hybrid beamforming-based transmitter structures for (a) fully connected, (b) partially-connected array of subarrays (AoSAs) and (c) partiallyconnected groups of subarrays (GoSAs) architectures.

the hardware complexity and power consumption, which is critical in $\mathrm{THz}$ systems.

In the proposed GoSA model, the RF precoder has unit-modulus constraints, i.e., $\left|\left[\mathbf{F}_{\mathrm{RF}}\right]_{i, j}\right|=\frac{1}{\sqrt{N_{\mathrm{T}}}}$ as $i \in$ $\left\{1, \ldots, N_{\mathrm{T}}\right\}$ and $j \in\left\{1, \ldots, N_{\mathrm{RF}}\right\}$, since $\mathbf{F}_{\mathrm{RF}}$ is constructed by using phase-shifters. Furthermore, we have power constrained $\left\|\mathbf{F}_{\mathrm{RF}} \mathbf{F}_{\mathrm{BB}}\right\|_{\mathcal{F}}=N_{\mathrm{S}}$. Thus, the $N_{\mathrm{T}} \times 1$ transmitted signal from the TX is given by $\mathbf{x}=\mathbf{F}_{\mathrm{RF}} \mathbf{F}_{\mathrm{BB}} \mathbf{s}$.

Assuming frequency-flat fading single-carrier transmission between the TX and RX [7], the received signal at the RX is given by

$$
\mathbf{y}=\sqrt{\rho} \mathbf{H F}_{\mathrm{RF}} \mathbf{F}_{\mathrm{BB}} \mathbf{s}+\mathbf{n},
$$

where $\mathbf{y} \in \mathbb{C}^{N_{\mathrm{R}}}$ is the output of $N_{\mathrm{R}}$ subarrayed antennas at the $\mathrm{RX}, \rho$ is the received power and $\mathbf{n} \in \mathbb{C}^{N_{\mathrm{R}}}$ denotes the additive white Gaussian noise (AWGN) vector with $\mathbf{n} \sim \mathcal{C N}\left(\mathbf{0}, \sigma_{n}^{2} \mathbf{I}_{N_{\mathrm{R}}}\right) . \mathbf{H} \in \mathbb{C}^{N_{\mathrm{R}} \times N_{\mathrm{T}}}$ denotes the $\mathrm{THz}$ channel matrix between the TX and the RX.

The structure of the $\mathrm{THz}$ channel matrix $\mathbf{H}$ is dominated by the LoS paths while the non-LoS channel components are small due to large reflection loses, scattering and refraction [7, 12]. In this work, we adopt the Saleh-Valenzuela (SV) $\mathrm{THz}$ channel model channel [7, 15], wherein $\mathbf{H}$ is constructed by the superposition of only LoS paths, i.e.,

$$
\mathbf{H}=\gamma \sum_{l=1}^{L} \alpha_{l} \mathbf{A}_{\mathrm{R}}\left(\Theta_{l}\right) \mathbf{A}_{\mathrm{T}}^{\mathrm{H}}\left(\Psi_{l}\right),
$$

where $\gamma=\sqrt{\frac{N_{\mathrm{T}} N_{\mathrm{R}}}{L}} . \alpha_{l} \in \mathbb{C}$ denotes the zero-mean Gaussian complex channel gain with variance $\sigma_{\alpha_{l}}^{2}$ corresponding to the $l$ th path. Similarly, $\Theta_{l}=\left\{\phi_{l}, \theta_{l}\right\}$ and $\Psi_{l}=\left\{\varphi_{l}, \vartheta_{l}\right\}$ denote the azimuth/elevation angle-of-arrival (AoA) and angleof-departure (AoD) of the received/transmitted paths at the RX and the TX, respectively. $\mathbf{A}_{\mathrm{R}}\left(\Theta_{l}\right) \in \mathbb{C}^{N_{\mathrm{R}} \times Q}$ and $\mathbf{A}_{\mathrm{T}}\left(\Psi_{l}\right) \in$ $\mathbb{C}^{N_{\mathrm{T}} \times Q}$ are the steering matrices corresponding to the AoAs and AoDs of the GoSAs, respectively, and they are defined as

$$
\mathbf{A}_{\mathrm{R}}\left(\Theta_{l}\right)=\left[\begin{array}{c}
\mathbf{a}_{\mathrm{R}, 1}^{\top}\left(\Theta_{l}\right) \\
\vdots \\
\mathbf{a}_{\mathrm{R}, N_{\mathrm{R}}}^{\top}\left(\Theta_{l}\right)
\end{array}\right], \mathbf{A}_{\mathrm{T}}\left(\Psi_{l}\right)=\left[\begin{array}{c}
\mathbf{a}_{\mathrm{T}, 1}^{\top}\left(\Psi_{l}\right) \\
\vdots \\
\mathbf{a}_{\mathrm{T}, N_{\mathrm{T}}}^{\top}\left(\Psi_{l}\right)
\end{array}\right]
$$

where $\mathbf{a}_{\mathrm{R}, n}\left(\Theta_{l}\right)\left(\mathbf{a}_{\mathrm{T}, m}\left(\Psi_{l}\right)\right)$ is $Q \times 1$ steering vector corresponding to the antennas in the $n$th $(m$ th) subarray for 
$n \in\left\{1 \ldots, N_{\mathrm{R}}\right\}\left(m \in\left\{1, \ldots, N_{\mathrm{T}}\right\}\right)$. The $i$ th element of the transmit steering vector $\mathbf{a}_{\mathrm{T}, m}\left(\Psi_{l}\right)$ is given by $\left[\mathbf{a}_{\mathrm{T}, m}\left(\Psi_{l}\right)\right]_{i}=$ $\frac{1}{\sqrt{N_{\mathrm{T}}}} \exp \left\{-\frac{2 \pi}{\lambda} \boldsymbol{\kappa}_{m, i}^{\top} \boldsymbol{\Omega}_{l}\right\}$, where $\lambda$ is the wavelength and $\boldsymbol{\kappa}_{m, i}=\left[x_{m, i}, y_{m, i}, z_{m, i}\right]^{\top}$ denotes the position of the $i$ th antenna of the $m$ th subarray in Cartesian coordinate system and $\boldsymbol{\Omega}_{l}$ is a direction-dependent parameter defined as $\boldsymbol{\Omega}_{l}=$ $\left[\cos \varphi_{l} \sin \vartheta_{l}, \sin \varphi_{l} \sin \vartheta_{l}, \cos \vartheta_{l}\right]^{\top}$. The structure of $\mathbf{a}_{\mathrm{R}}\left(\Theta_{l}\right)$ is similar to that of $\mathbf{a}_{\mathrm{T}}\left(\Psi_{l}\right)$. Without loss of generality, we assume that the antennas are perfectly calibrated against mutual coupling and gain/phase mismatches. Then, the $(n, m)$ th element of $\mathbf{H}$ is given by

$$
[\mathbf{H}]_{n, m}=\gamma \sum_{l=1}^{L} \alpha_{l} \mathbf{a}_{\mathrm{R}, n}\left(\Theta_{l}\right) \odot \mathbf{a}_{\mathrm{T}, m}^{*}\left(\Psi_{l}\right) .
$$

By connecting the $Q$ antennas in the subarrays to a single phase-shifter, we are able to construct an $N_{\mathrm{T}} \times N_{\mathrm{RF}}$, (instead of $N_{\mathrm{T}} Q \times N_{\mathrm{RF}}$ as in Fig. 2a and Fig. 2b) RF precoder, as illustrated in Fig. 2c. Using partially-connected GoSA, the associated RF precoder has the form of

$$
\mathbf{F}_{\mathrm{RF}}=\left[\begin{array}{cccc}
\mathbf{u}_{1} & \mathbf{0} & \cdots & \mathbf{0} \\
\mathbf{0} & \mathbf{u}_{2} & \cdots & \mathbf{0} \\
\vdots & \mathbf{0} & \ddots & \mathbf{0} \\
\mathbf{0} & \mathbf{0} & \cdots & \mathbf{u}_{N_{\mathrm{RF}}}
\end{array}\right] \in \mathbb{C}^{N_{\mathrm{T}} \times N_{\mathrm{RF}}},
$$

where $\mathbf{u}_{i} \in \mathbb{C}^{\bar{N}}$ represents a portion $N_{\mathrm{T}} \times 1$ phase-shifter values with indices $\{(i-1) \bar{N}+1, \ldots, i \bar{N}\}$ for $i \in\left\{1, \ldots, N_{\mathrm{RF}}\right\}$, where $\bar{N}=\frac{N_{\mathrm{T}}}{N_{\mathrm{RF}}}$. Each entry of $\mathbf{u}_{i}$ is then applied to $Q$ antennas in $N_{\mathrm{T}}$ subarrays to steer the transmitted beams (see, e.g., Fig. 2c) so that a total of $N_{\mathrm{T}} Q$ antennas are fed.

To address the performance degradation due to GoSA, the columns of $\mathbf{F}_{\mathrm{RF}}$ is designed with overlapping terms [16]. Assume $\overline{\mathbf{u}}_{i} \in \mathbb{C}^{\bar{M}}$ to include the overlapped phase-shifter terms, where $\bar{M} \in\left[\bar{N}, N_{\mathrm{T}}-N_{\mathrm{RF}}+1\right]$, for which $\bar{M}=$ $\bar{N}$ provides non-overlapped PC structure as in (5) while $\bar{M}=N_{\mathrm{T}}-N_{\mathrm{RF}}+1$ provides maximum overlap among the phase-shifters. In this case, the performance improvement is at the cost of using more phase-shifters. Nevertheless, it still has lower number of phase-shifters as compared to the partially non-overlapped case in conventional AoSA. The use of PC/PCO GoSA structure provides higher DoF as compared to the simple phased-array MIMO radar structure, for which $N_{\mathrm{RF}}=1$ and we have a fully-connected MIMO structure when $N_{\mathrm{RF}}=N_{\mathrm{T}}$. While MIMO radar outperforms the phased-array in terms of angular resolution and DoF for parameter estimation and parameter identification, phasearray provides higher coherent processing gain and lower computation and hardware complexity [17]. This complexity is further reduced by using the GoSA structure by feeding each of $Q$ antennas with the same phase shift. Thus, the partiallyconnected GoSA provides a trade-off between the DoF and the hardware complexity, both of which increase as $N_{\mathrm{RF}} \rightarrow N_{\mathrm{T}}$.

In communications-only systems, the aim is to design the hybrid precoders such that the spectral efficiency at the TX is maximized [14, 18, 19], while there are also other related performance metrics, such as energy-efficiency and minimum mean-squared-error (MMSE). By decoupling the beamformer design problem at the TX and the RX, the mutual information at the TX is maximized instead of spectral efficiency, for which a perfect combiner is assumed at the receiver [19]. Once the transmitter is designed, the receive beamforming design is done by using the MMSE as performance metric as in [14, $18,19]$. Then the mutual information of the communications system is given by

$\mathcal{I}\left(\mathbf{F}_{\mathrm{RF}}, \mathbf{F}_{\mathrm{BB}}\right)=\log _{2}\left|\mathbf{I}_{N_{\mathrm{R}}}+\frac{\rho}{N_{\mathrm{S}} \sigma_{n}^{2}} \mathbf{H} \mathbf{F}_{\mathrm{RF}} \mathbf{F}_{\mathrm{BB}} \mathbf{F}_{\mathrm{BB}}^{\mathrm{H}} \mathbf{F}_{\mathrm{RF}}^{\mathrm{H}} \mathbf{H}^{\mathrm{H}}\right|$.

We note here that the maximization of (6) is provided by exploiting the similarity between the hybrid beamformer $\mathbf{F}_{\mathrm{RF}} \mathbf{F}_{\mathrm{BB}}$ and the optimal unconstrained beamformer $\mathbf{F}_{\mathrm{C}} \in$ $\mathbb{C}^{N_{\mathrm{T}} \times N_{\mathrm{S}}}$. The latter is obtained from the right singular matrix of the channel matrix $\mathbf{H}[14,19]$. The singular value decomposition of the channel matrix is $\mathbf{H}=\mathbf{U}_{\mathbf{H}} \boldsymbol{\Pi} \mathbf{V}_{\mathbf{H}}^{\mathrm{H}}$, where $\mathbf{U}_{\mathbf{H}} \in \mathbb{C}^{N_{\mathrm{R}} \times \operatorname{rank}(\mathbf{H})}$ and $\mathbf{V}_{\mathbf{H}} \in \mathbb{C}^{N_{\mathrm{T}} \times \operatorname{rank}(\mathbf{H})}$ are the left and the right singular value matrices of the channel matrix, respectively, and $\boldsymbol{\Pi}$ is $\operatorname{rank}(\mathbf{H}) \times \operatorname{rank}(\mathbf{H})$ matrix composed of the singular values of $\mathbf{H}$ in descending order. By decomposing $\boldsymbol{\Pi}$ and $\mathbf{V}_{\mathbf{H}}$ as $\boldsymbol{\Pi}=\operatorname{diag}\{\widetilde{\boldsymbol{\Pi}}, \overline{\boldsymbol{\Pi}}\}, \mathbf{V}_{\mathbf{H}}=\left[\widetilde{\mathbf{V}}_{\mathbf{H}}, \overline{\mathbf{V}}_{\mathbf{H}}\right]$, where $\widetilde{\mathbf{V}}_{\mathbf{H}} \in \mathbb{C}^{N_{\mathrm{T}} \times N_{\mathrm{S}}}$, the unconstrained precoder is readily obtained as $\mathbf{F}_{\mathrm{C}}=\widetilde{\mathbf{V}}_{\mathbf{H}}$ [19]. Then, the maximization of (6) is achieved by minimizing the Euclidean distance between $\mathbf{F}_{\mathrm{C}}$ and $\mathbf{F}_{\mathrm{RF}} \mathbf{F}_{\mathrm{BB}}$ as

$$
\begin{aligned}
\min _{\mathbf{F}_{\mathrm{RF}}, \mathbf{F}_{\mathrm{BB}}} & \left\|\mathbf{F}_{\mathrm{RF}} \mathbf{F}_{\mathrm{BB}}-\mathbf{F}_{\mathrm{C}}\right\|_{\mathcal{F}} \\
\text { s. t. : } & \left\|\mathbf{F}_{\mathrm{RF}} \mathbf{F}_{\mathrm{BB}}\right\|_{\mathcal{F}}=N_{\mathrm{S}}, \\
& \left|\left[\mathbf{F}_{\mathrm{RF}}\right]_{i, j}\right|=\frac{1}{\sqrt{N_{\mathrm{T}}}}, \forall i, j .
\end{aligned}
$$

The goal of radar processing is to achieve the highest possible SNR gain towards the direction of interest. The radar first transmits an omni-directional waveforms to detect the unknown targets within the angular space of interest in the search phase, then it generates directional beams towards to the targets for tracking purposes [17]. We assume a subarrayed MIMO radar architecture with GoSAs, wherein each GoSA is used to coherently transmit waveforms that are orthogonal to the ones generated by other GoSAs, thereby, coherent processing gain is achieved. Denote $\left\{\Phi_{1}, \ldots, \Phi_{K}\right\}$ as the set of target directions $\left(\Phi_{k}=\left(\bar{\varphi}_{k}, \bar{\vartheta}_{k}\right)\right)$, then, the $N_{\mathrm{T}} \times K$ GoSA-MIMO radar-only beamformer is modeled as $\mathbf{F}_{\mathrm{R}}=$ blkdiag $\left\{\mathbf{v}_{1}, \ldots, \mathbf{v}_{K}\right\}$ similar to (5), where $\mathbf{v}_{k} \in \mathbb{C}^{\bar{K}}$ denotes the values of the transmit steering vector $\mathbf{a}_{\mathrm{T}}\left(\Phi_{k}\right) \in \mathbb{C}^{N_{\mathrm{T}}}$ with indices $\{(k-1) \bar{K}+1, \ldots, k \bar{K}\}$ for $k=1 \ldots, K$ and $\bar{K}=\frac{N_{\mathrm{T}}}{K}$.It is possible to construct $\mathbf{F}_{\mathrm{R}}$ via overlapped GoSA with $\overline{\mathbf{v}}_{k} \in \mathbb{C}^{N_{\mathrm{T}}-K+1}$ for $k \in\{1, \ldots, K\}$.

The estimation of the target directions $\left\{\Phi_{k}\right\}_{k=1}^{K}$ is performed in the search phase of the radar. In this work, we assume that search operation is completed and the direction information of the targets is acquired prior to the beamformer design. The beampattern of the radar with GoSA structure is $B(\tilde{\Phi})=\operatorname{Trace}\left\{\mathbf{A}_{\mathrm{T}}^{\mathrm{H}}(\tilde{\Phi}) \mathbf{R} \mathbf{A}_{\mathrm{T}}(\tilde{\Phi})\right\}$, where $\mathbf{R} \in \mathbb{C}^{N_{\mathrm{T}} \times N_{\mathrm{T}}}$ 
is the covariance matrix of the transmitted signal, then the design of the radar beampattern is equivalent to the design of the covariance matrix of the radar probing signals subject to the hybrid architecture of the beamformers. In case of a single target scenario, the optimal beamformer is known to be conventional nonadaptive beamformer, i.e., steering vector corresponding to the direction of interest [16]. When there are multiple targets, the covariance matrix of the transmitted signal is utilized. In case of multiple targets in radar-only scenario with hybrid beamforming, we define the covariance matrix of the transmitted signal $\mathrm{x}$ as

$$
\begin{aligned}
\mathbf{R} & =\mathbb{E}\left\{\mathbf{x x}^{\mathrm{H}}\right\}=\mathbb{E}\left\{\mathbf{F}_{\mathrm{RF}} \mathbf{F}_{\mathrm{BB}} \mathbf{s s}^{\mathrm{H}} \mathbf{F}_{\mathrm{BB}}^{\mathrm{H}} \mathbf{F}_{\mathrm{RF}}^{\mathrm{H}}\right\}, \\
& =\mathbf{F}_{\mathrm{RF}} \mathbf{F}_{\mathrm{BB}} \mathbb{E}\left\{\mathbf{s s}^{\mathrm{H}}\right\} \mathbf{F}_{\mathrm{BB}}^{\mathrm{H}} \mathbf{F}_{\mathrm{RF}}^{\mathrm{H}}, \\
& =\mathbf{F}_{\mathrm{RF}} \mathbf{F}_{\mathrm{BB}} \mathbf{F}_{\mathrm{BB}}^{\mathrm{H}} \mathbf{F}_{\mathrm{RF}}^{\mathrm{H}},
\end{aligned}
$$

which requires the design of hybrid beamformers $\mathbf{F}_{\mathrm{RF}}, \mathbf{F}_{\mathrm{BB}}$. The hybrid beamformer design problem for radar-only system is solved by minimizing the Euclidean distance between $\mathbf{F}_{\mathrm{RF}} \mathbf{F}_{\mathrm{BB}}$ and $\mathbf{F}_{\mathrm{R}} \mathbf{P}$ as

$$
\begin{aligned}
\min _{\mathbf{F}_{\mathrm{RF}}, \mathbf{F}_{\mathrm{BB}}, \mathbf{P}} & \left\|\mathbf{F}_{\mathrm{RF}} \mathbf{F}_{\mathrm{BB}}-\mathbf{F}_{\mathrm{R}} \mathbf{P}\right\|_{\mathcal{F}} \\
\text { s. t. : } & \left\|\mathbf{F}_{\mathrm{RF}} \mathbf{F}_{\mathrm{BB}}\right\|_{\mathcal{F}}=N_{\mathrm{S}}, \\
& \left|\left[\mathbf{F}_{\mathrm{RF}}\right]_{i, j}\right|=\frac{1}{\sqrt{N_{\mathrm{T}}}}, \forall i, j, \quad \mathbf{P} \mathbf{P}^{\mathrm{H}}=\mathbf{I}_{N_{\mathrm{S}}},
\end{aligned}
$$

where the unitary matrix $\mathbf{P} \in \mathbb{C}^{K \times N_{\mathrm{S}}}$ is an auxiliary variable to provide a change of dimension between $\mathbf{F}_{\mathrm{RF}} \mathbf{F}_{\mathrm{BB}}$ and $\mathbf{F}_{\mathrm{R}}$ without causing any distortion in the radar beampattern and $\mathbf{P} \mathbf{P}^{\mathrm{H}}=\mathbf{I}_{K}$.

The aim of this work is designing the hybrid beamformer $\mathbf{F}_{\mathrm{RF}} \mathbf{F}_{\mathrm{BB}}$ to simultaneously maximize the spectral efficiency of the communications link and provide as much SNR as possible towards the radar targets by forming the beampattern of the transmit antenna array. To jointly solve the problems in (7) and (9), we formulate the following problem,

$$
\min _{\mathbf{F}_{\mathrm{RF}}, \mathbf{F}_{\mathrm{BB}}, \mathbf{P}} \eta\left\|\mathbf{F}_{\mathrm{RF}} \mathbf{F}_{\mathrm{BB}}-\mathbf{F}_{\mathrm{C}}\right\|_{\mathcal{F}}+(1-\eta)\left\|\mathbf{F}_{\mathrm{RF}} \mathbf{F}_{\mathrm{BB}}-\mathbf{F}_{\mathrm{R}} \mathbf{P}\right\|_{\mathcal{F}}
$$$$
\text { s. t.: }\left\|\mathbf{F}_{\mathrm{RF}} \mathbf{F}_{\mathrm{BB}}\right\|_{\mathcal{F}}=N_{\mathrm{S}} \text {, }
$$

$$
\begin{aligned}
& \left|\left[\mathbf{F}_{\mathrm{RF}}\right]_{i, j}\right|=\frac{1}{\sqrt{N_{\mathrm{T}}}}, \forall i, j \in \mathcal{S}, \\
& \left|\left[\mathbf{F}_{\mathrm{RF}}\right]_{i, j}\right|=0, \quad \forall i, j \in \overline{\mathcal{S}}, \quad \mathbf{P P}^{\mathrm{H}}=\mathbf{I}_{N_{\mathrm{S}}},
\end{aligned}
$$

where $\mathcal{S}$ and $\overline{\mathcal{S}}$ denotes the set of non-zero and zero terms in $\mathbf{F}_{\mathrm{RF}}$ due to overlapped structure in (5), respectively. In (10), $0 \leq \eta \leq 1$ provides the trade-off between the radar and communications tasks. If $\eta=1(\eta=0)$, (10) corresponds to communications-only (radar-only) beamformer design problem. The optimization problem (10) is difficult solve because of several matrix variables $\mathbf{F}_{\mathrm{RF}}, \mathbf{F}_{\mathrm{BB}}, \mathbf{P}$, and nonconvex constraints. A common approach is to use alternating techniques, i.e., estimating the unknown variables one-byone while fixing the others. While this approach does not guarantee the optimality, its convergence is proved in the relevant literature, e.g., $[14,18,20]$.

\section{HyBRid BEAMFORMER DESIGN}

Denote $f\left(\mathbf{F}_{\mathrm{RF}}, \mathbf{F}_{\mathrm{BB}}, \mathbf{P}\right)$ as the cost function in (10), which is rewritten as

$$
\begin{aligned}
& f\left(\mathbf{F}_{\mathrm{RF}}, \mathbf{F}_{\mathrm{BB}}, \mathbf{P}\right)= \\
& \left\|\eta\left[\mathbf{F}_{\mathrm{RF}} \mathbf{F}_{\mathrm{BB}}-\mathbf{F}_{\mathrm{C}}\right]\right\|_{\mathcal{F}}+\left\|(1-\eta)\left[\mathbf{F}_{\mathrm{RF}} \mathbf{F}_{\mathrm{BB}}-\mathbf{F}_{\mathrm{R}} \mathbf{P}\right]\right\|_{\mathcal{F}} .
\end{aligned}
$$

Then, using triangle inequality, we get

$$
\begin{aligned}
& f\left(\mathbf{F}_{\mathrm{RF}}, \mathbf{F}_{\mathrm{BB}}, \mathbf{P}\right) \geq \\
& \left\|\eta \mathbf{F}_{\mathrm{RF}} \mathbf{F}_{\mathrm{BB}}-\eta \mathbf{F}_{\mathrm{C}}+(1-\eta) \mathbf{F}_{\mathrm{RF}} \mathbf{F}_{\mathrm{BB}}-(1-\eta) \mathbf{F}_{\mathrm{R}} \mathbf{P}\right\|_{\mathcal{F}} \\
& =\left\|\mathbf{F}_{\mathrm{RF}} \mathbf{F}_{\mathrm{BB}}-\eta \mathbf{F}_{\mathrm{C}}-(1-\eta) \mathbf{F}_{\mathrm{R}} \mathbf{P}\right\|_{\mathcal{F}} .
\end{aligned}
$$

Define $\mathbf{F}_{\mathrm{CR}} \in \mathbb{C}^{N_{\mathrm{T}} \times N_{\mathrm{S}}}$ as the JRC beamformer as

$$
\mathbf{F}_{\mathrm{CR}}=\eta \mathbf{F}_{\mathrm{C}}+(1-\eta) \mathbf{F}_{\mathrm{R}} \mathbf{P},
$$

and define the new cost function $\bar{f}\left(\mathbf{F}_{\mathrm{RF}}, \mathbf{F}_{\mathrm{BB}}, \mathbf{P}\right)$ as

$$
\bar{f}\left(\mathbf{F}_{\mathrm{RF}}, \mathbf{F}_{\mathrm{BB}}, \mathbf{P}\right)=\left\|\mathbf{F}_{\mathrm{RF}} \mathbf{F}_{\mathrm{BB}}-\mathbf{F}_{\mathrm{CR}}\right\|_{\mathcal{F}},
$$

where we have $\bar{f}\left(\mathbf{F}_{\mathrm{RF}}, \mathbf{F}_{\mathrm{BB}}, \mathbf{P}\right) \leq f\left(\mathbf{F}_{\mathrm{RF}}, \mathbf{F}_{\mathrm{BB}}, \mathbf{P}\right)$ due to (12). Then, we rewrite the optimization problem (10) in a compact form as

$$
\begin{array}{ll}
\min _{\mathbf{F}_{\mathrm{RF}}, \mathbf{F}_{\mathrm{BB}}, \mathbf{P}}\left\|\mathbf{F}_{\mathrm{RF}} \mathbf{F}_{\mathrm{BB}}-\mathbf{F}_{\mathrm{CR}}\right\|_{\mathcal{F}} \\
\text { s. t. : } & \left\|\mathbf{F}_{\mathrm{RF}} \mathbf{F}_{\mathrm{BB}}\right\|_{\mathcal{F}}=N_{\mathrm{S}}, \\
& \left|\left[\mathbf{F}_{\mathrm{RF}}\right]_{i, j}\right|=\frac{1}{\sqrt{N_{\mathrm{T}}}}, \forall i, j \in \mathcal{S}, \\
& \left|\left[\mathbf{F}_{\mathrm{RF}}\right]_{i, j}\right|=0, \quad \forall i, j \in \overline{\mathcal{S}}, \\
& \mathbf{P} \mathbf{P}^{\mathrm{H}}=\mathbf{I}_{N_{\mathrm{S}}} .
\end{array}
$$

Now, the problem (15) looks similar to the communicationsonly problem in (7), and is solved via alternating minimization techniques suggested to solve (7), e.g., [14, 19]. In this case, $\mathbf{F}_{\mathrm{RF}}, \mathbf{F}_{\mathrm{BB}}$ and $\mathbf{P}$ are estimated one-by-one while the others are fixed. By fixing $\mathbf{F}_{\mathrm{RF}}$ and $\mathbf{F}_{\mathrm{BB}}, \mathbf{P}$ is found via the SVD of the matrix $\mathbf{F}_{\mathrm{R}}^{\mathrm{H}} \mathbf{F}_{\mathrm{RF}} \mathbf{F}_{\mathrm{BB}}$, i.e.,

$$
\mathbf{P}=\widetilde{\mathbf{U}} \mathbf{I}_{K \times N_{\mathrm{S}}} \tilde{\mathbf{V}},
$$

for which we have $\widetilde{\mathbf{U}} \boldsymbol{\Sigma} \widetilde{\mathbf{V}}^{\mathrm{H}}=\mathbf{F}_{\mathrm{R}}^{\mathrm{H}} \mathbf{F}_{\mathrm{RF}} \mathbf{F}_{\mathrm{BB}}$ and $\mathbf{I}_{K \times N_{\mathrm{S}}}=$ $\left[\mathbf{I}_{K}, \mathbf{0}_{K \times\left(N_{\mathrm{S}}-K\right)}\right]$. Similarly, when $\mathbf{F}_{\mathrm{RF}}$ and $\mathbf{P}$ are fixed, $\mathbf{F}_{\mathrm{BB}}$ is calculated as $\mathbf{F}_{\mathrm{BB}}=\mathbf{F}_{\mathrm{RF}}^{\dagger} \mathbf{F}_{\mathrm{CR}}$, and normalized as $\mathbf{F}_{\mathrm{BB}}=$ $\frac{\sqrt{N_{\mathrm{S}}}}{\left\|\mathbf{F}_{\mathrm{RF}} \mathbf{F}_{\mathrm{BB}}\right\|_{\mathcal{F}}} \mathbf{F}_{\mathrm{BB}}$.

The main challenge in (15) is the estimation of $\mathbf{F}_{\mathrm{RF}}$ due to unit-modulus constraints. In FC case, $\mathbf{F}_{\mathrm{RF}}$ is found via MObased techniques and the optimal solution is readily obtained for PC structure via phase-rotation $[14,20]$. However, the design of $\mathbf{F}_{\mathrm{RF}}$ for the overlapped case is not straightforward due to the constraint (15c). Thus, we propose a MMO-based solution to account for (15c) in the following.

Assume that $\mathbf{F}_{\mathrm{BB}}$ and $\mathbf{P}$ are fixed, then (15) is written in vectorized form as

$$
\begin{array}{cl}
\min _{\mathbf{f}_{\mathrm{RF}}} & \left\|\mathbf{G}_{\mathrm{RF}}-\mathbf{f}_{\mathrm{CR}}\right\|_{\mathcal{F}} \\
\text { s. t. : } & \left|\left[\mathbf{f}_{\mathrm{RF}}\right]_{i}\right|=\frac{1}{\sqrt{N_{\mathrm{T}}}}, \quad \forall i \in \mathcal{V}, \\
& \left|\left[\mathbf{f}_{\mathrm{RF}}\right]_{i}\right|=0, \quad \forall i \in \overline{\mathcal{V}},
\end{array}
$$



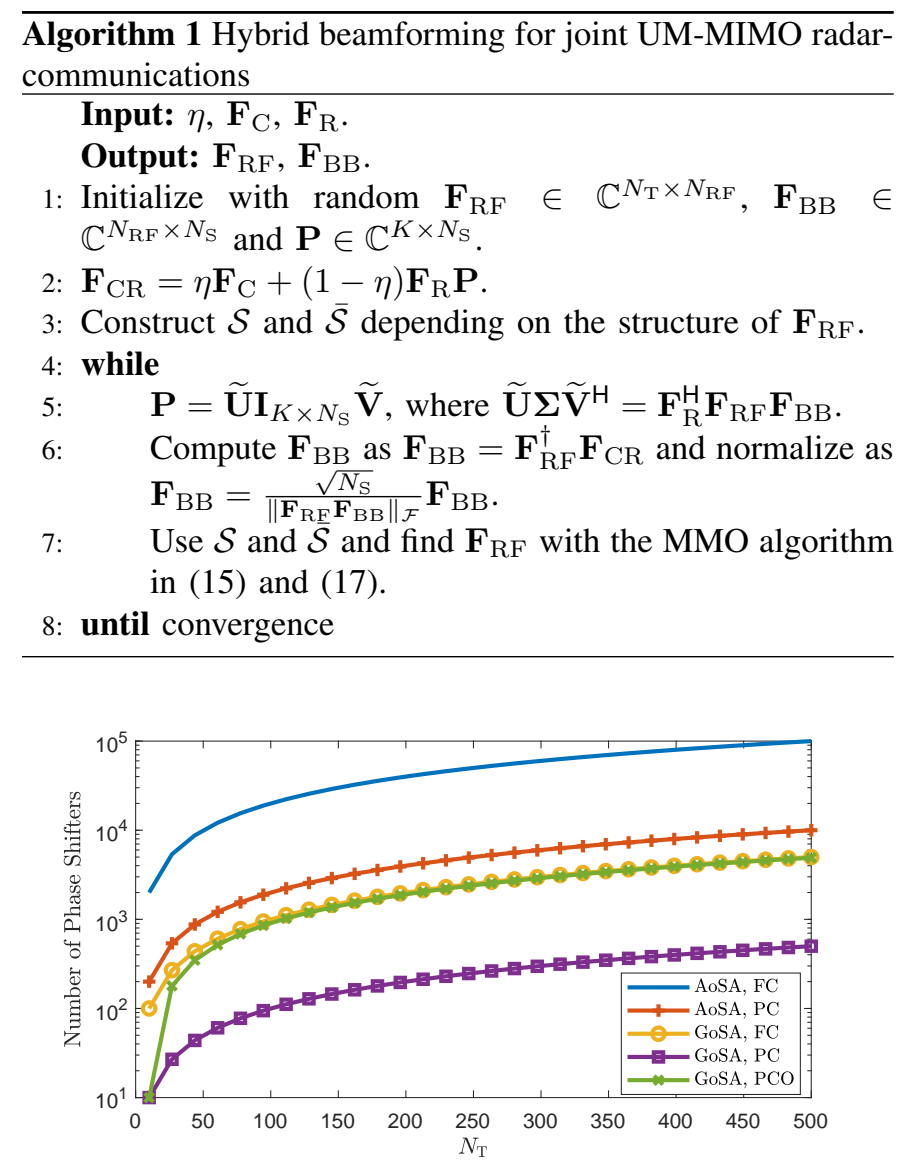

Fig. 3. Number of phase-shifters versus $N_{\mathrm{T}}$ when $Q=20$ for $N_{\mathrm{RF}}=10$.

where $\mathbf{G}=\left(\mathbf{F}_{\mathrm{BB}}^{\mathrm{T}}\right) \otimes \mathbf{I}_{N_{\mathrm{T}}} \in \mathbb{C}^{N_{\mathrm{T}} N_{\mathrm{S}} \times N_{\mathrm{T}} N_{\mathrm{RF}}}, \mathbf{f}_{\mathrm{RF}}=$ $\operatorname{vec}\left\{\mathbf{F}_{\mathrm{RF}}\right\} \in \mathbb{C}^{N_{\mathrm{T}} N_{\mathrm{RF}}}$ and $\mathbf{f}_{\mathrm{CR}}=\operatorname{vec}\left\{\mathbf{F}_{\mathrm{CR}}\right\} \in \mathbb{C}^{N_{\mathrm{T}} N_{\mathrm{S}}} \cdot \mathcal{V}$ and $\overline{\mathcal{V}}$ denote the set of non-zero and zero terms in $\mathbf{f}_{\mathrm{RF}}$, respectively. The sizes of $\mathcal{V}$ and $\overline{\mathcal{V}}$ depend on the selection of $\bar{M}$. As an example, for $N_{\mathrm{T}}=100, N_{\mathrm{RF}}=10$ and $\bar{M}=N_{\mathrm{RF}}\left(N_{\mathrm{T}}-N_{\mathrm{RF}}+1\right)$, we have $|\mathcal{V}|=910$ and $|\overline{\mathcal{V}}|=90$. Now, the aim is to exclude the portion of $\mathbf{G}$ and $\mathbf{f}_{\mathrm{RF}}$ corresponding to $\overline{\mathcal{V}}$ and find the portion of $\mathbf{f}_{\mathrm{RF}}$ corresponding to $\mathcal{V}$ so that we employ MO accordingly and all the elements of the unknown vector will obey unit-modulus constraints. We present the algorithmic steps of the proposed method in Algorithm 1.

\section{NUMERICAL EXPERIMENTS}

In this section, we evaluate the performance of the proposed hybrid beamforming approach for different array structures. The communications performance of the algorithms is evaluated in terms of spectral efficiency while the radar performance is presented with the beampattern analysis of the hybrid beamformers. Furthermore, we analyze the trade-off between both tasks by sweeping $\eta$ for $[0,1]$. The hybrid beamformers are designed for FC, PC and PCO array structures. The proposed MMO-based approach is used to design PCO array. Then, it is compared with the PC and FC arrays, which employ

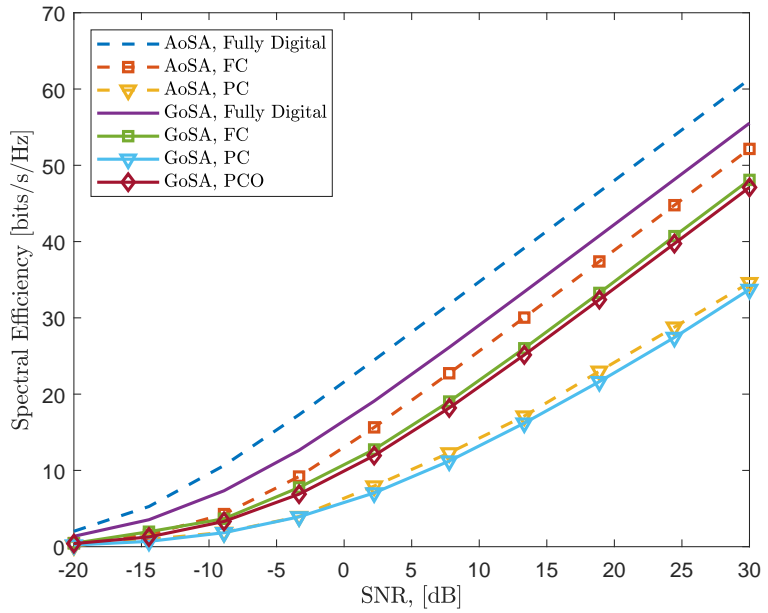

Fig. 4. Spectral efficiency versus SNR for hybrid beamforming when $\eta=0.5$.

the MO-based alternating minimization (MO-AltMin) [14] and triple AltMin (TAltMin) approach in [20], respectively, while the fully digital unconstrained beamformers are used as a benchmark [19].

In the simulations, unless stated otherwise, we select the operating frequency as $f_{c}=300 \mathrm{GHz}$, which is in low- THz band $(100 \mathrm{GHz}-1 \mathrm{THz})$ and applicable for long range radar (LRR) $(\sim 150 \mathrm{~m})$ [21]. We also select $\Delta=\Delta_{\mathrm{x}}=\Delta_{\mathrm{y}}=$ $\lambda / 2$ and $\delta=\delta_{\mathrm{x}}=\delta_{\mathrm{y}}=\lambda / 4$. At the TX and $\mathrm{RX}, N_{\mathrm{T}_{\mathrm{x}}}=$ $N_{\mathrm{T}_{\mathrm{y}}}=32\left(N_{\mathrm{T}}=1024\right)$ and $N_{\mathrm{R}_{\mathrm{x}}}=N_{\mathrm{R}_{\mathrm{y}}}=9\left(N_{\mathrm{R}}=81\right)$ subarrays are used, respectively, with $Q_{\mathrm{x}}=Q_{\mathrm{y}}=3(Q=9)$. Thus, the resultant architecture forms a $729 \times 9216$ UM-MIMO transceiver. We assume that $N_{\mathrm{RF}}=16 \mathrm{RF}$ chains are used at the TX to transmit $N_{\mathrm{S}}=4$ data streams to the RX via the THz channel which is assumed to include $L=5$ paths, where $\phi_{l}, \varphi_{l} \in\left[-150^{\circ}, 150^{\circ}\right]$ and $\theta_{l}, \vartheta_{l} \in\left[70^{\circ}, 90^{\circ}\right]$. The TX simultaneously generates beams towards both $\mathrm{RX}$ and $K=3$ radar targets located at $\left\{\left(60^{\circ}, 70^{\circ}\right),\left(110^{\circ}, 75^{\circ}\right),\left(140^{\circ}, 80^{\circ}\right)\right\}$.

Fig. 3 shows the number of phase-shifters with respect to $N_{\mathrm{T}}$ for different array structures, i.e., AoSA and GoSA. The FC structures employ $N_{\mathrm{T}} Q N_{\mathrm{RF}}$ and $N_{\mathrm{T}} N_{\mathrm{RF}}$ phase-shifters for AoSA and GoSA, respectively, while the PC structures are more efficient since only $N_{\mathrm{T}} Q$ and $N_{\mathrm{T}}$ phase-shifters are used for AoSA and GoSA. Compared to AoSA, the proposed GoSA structure employs much less phase-shifters than that of AoSA for $Q \geq N_{\mathrm{RF}}$ and they become equal if $Q=1$. Thus, GoSA is much more energy-efficient than AoSA.

Fig. 4 shows the spectral efficiency with respect to SNR for hybrid beamforming when $\eta=0.5$. We observe that GoSA performs slightly lower than AoSA structure while using $Q=9$ times less phase-shifters, which significantly lowers the hardware complexity of UM-MIMO system. While PC structures have the lowest hardware complexities, they perform the worst as compared to FC case. The GoSA with PCO improves the spectral efficiency by employing relatively 


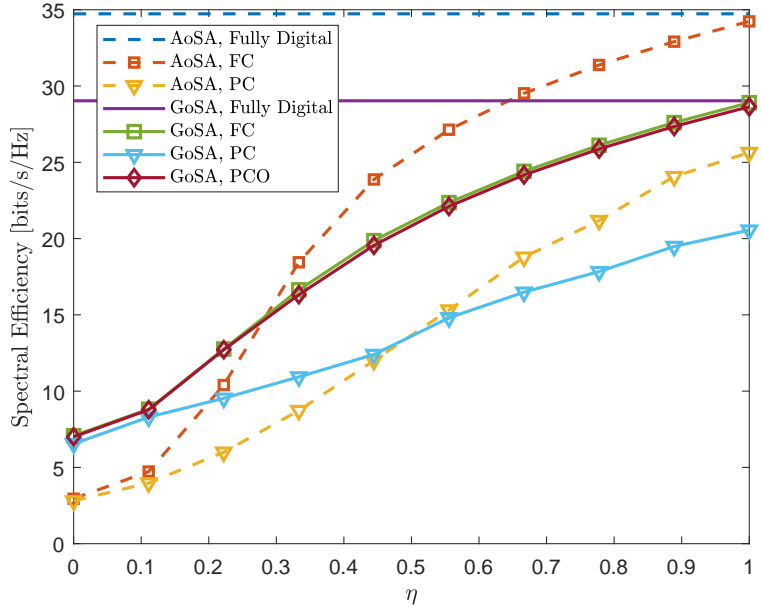

Fig. 5. Spectral efficiency versus $\eta$ for $\mathrm{SNR}=10 \mathrm{~dB}$.

more phase-shifters which still less than that of AoSA. The gap between the unconstrained (fully digital) and hybrid beamformers is large due to the trade-off between radar and communications tasks with $\eta=0.5$.

In Fig. 5, the spectral efficiency is presented with respect to $\eta$, wherein we note that as $\eta \rightarrow 1$, the spectral efficiency for the FC, PC and PCO approaches to the performance of unconstrained beamformer, i.e., $\mathbf{F}_{\mathrm{C}}$. When $\eta \rightarrow 0$, then the RF precoder $\mathbf{F}_{\mathrm{RF}}$ generates the beams towards the radar targets only, thus the spectral efficiency is reduced. As a result, the selection of $\eta$ is critical. In practice, $\eta$ is increased if the communications task is more critical than tracking the targets or when there is no target. Conversely, lower $\eta$ is selected if the radar task demands more resources, e.g., more transmit power is required depending on the range of the radar targets.

\section{CONCLUSIONS}

In this paper, we introduced THz UM-MIMO JRC architecture for hybrid beamforming. To lower the hardware complexity critical in THz systems, we proposed GoSA UMMIMO architecture. We developed hybrid beamforming via PCO structures to provide a trade-off between higher spectral efficiency and hardware complexity in terms of the number of phase-shifters. We evaluated the performance of the proposed methods in terms of spectral efficiency and radar beampattern. We demonstrated that GoSA provides less hardware complexity compared to full array and AoSA structures.

\section{ACKNOWLEDGMENT}

This work was supported in part by the ERC Project AGNOSTIC.

\section{REFERENCES}

[1] R. W. Heath, N. Gonzalez-Prelcic, S. Rangan, W. Roh, and A. M. Sayeed, "An overview of signal processing techniques for millimeter wave MIMO systems," IEEE J. Sel. Topics Signal Process., vol. 10, no. 3, pp. 436-453, 2016.
[2] K. V. Mishra, M. R. Bhavani Shankar, V. Koivunen, B. Ottersten, and S. A. Vorobyov, "Toward millimeter wave joint radar-communications: A signal processing perspective," IEEE Signal Process. Mag., vol. 36, no. 5, pp. 100-114, 2019.

[3] H.-J. Song and T. Nagatsuma, "Present and future of terahertz communications," IEEE Trans. THz Sci. Technol., vol. 1, no. 1, pp. 256-263, 2011.

[4] H. Sarieddeen, M.-S. Alouini, and T. Y. Al-Naffouri, "An overview of signal processing techniques for terahertz communications," arXiv, May 2020.

[5] L. Ju, B. Geng, J. Horng, C. Girit, M. Martin, Z. Hao, H. A. Bechtel, X. Liang, A. Zettl, Y. R. Shen et al., "Graphene plasmonics for tunable terahertz metamaterials," Nature nanotechnology, vol. 6, no. 10, pp. 630634, 2011.

[6] M. Moccia, C. Koral, G. P. Papari, S. Liu, L. Zhang, R. Y. Wu, G. Castaldi, T. J. Cui, V. Galdi, and A. Andreone, "Suboptimal coding metasurfaces for terahertz diffuse scattering," Scientific reports, vol. 8, no. 1, pp. 1-9, 2018

[7] H. Sarieddeen, M.-S. Alouini, and T. Y. Al-Naffouri, "Terahertz-band ultra-massive spatial modulation MIMO," IEEE J. Sel. Areas Commun., vol. 37, no. 9, pp. 2040-2052, 2019.

[8] C. Chaccour, M. N. Soorki, W. Saad, M. Bennis, P. Popovski, and M. Debbah, "Seven defining features of terahertz $(\mathrm{THz})$ wireless systems: A fellowship of communication and sensing," arXiv preprint arXiv:2102.07668, 2021.

[9] J. Liu, K. V. Mishra, and M. Saquib, "Co-designing statistical MIMO radar and in-band full-duplex multi-user MIMO communications," arXiv preprint arXiv:2006.14774, 2020.

[10] A. Alkhateeb, O. E. Ayach, G. Leus, and R. W. Heath, "Hybrid precoding for millimeter wave cellular systems with partial channel knowledge," in IEEE Inf. Th. Appl. Workshop, 2013, pp. 1-5.

[11] B. Ning, Z. Chen, W. Chen, Y. Du, and J. Fang, "Terahertz multi-user massive MIMO with intelligent reflecting surface: Beam training and hybrid beamforming," IEEE Trans. Veh. Technol., 2021, in press.

[12] C. Lin and G. Y. L. Li, "Terahertz Communications: An Array-ofSubarrays Solution," IEEE Commun. Mag., vol. 54, no. 12, pp. 124-131, Dec 2016

[13] C. Han, J. M. Jornet, and I. Akyildiz, "Ultra-Massive MIMO Channel Modeling for Graphene-Enabled Terahertz-Band Communications," 2018 IEEE 87th Vehicular Technology Conference (VTC Spring), pp. $1-5$, Jun 2018.

[14] X. Yu, J. Shen, J. Zhang, and K. B. Letaief, "Alternating Minimization Algorithms for Hybrid Precoding in Millimeter Wave MIMO Systems," IEEE J. Sel. Topics Signal Process., vol. 10, no. 3, pp. 485-500, April 2016.

[15] H. Yuan, N. Yang, K. Yang, C. Han, and J. An, "Hybrid Beamforming for Terahertz Multi-Carrier Systems Over Frequency Selective Fading," IEEE Trans. Commun., vol. 68, no. 10, pp. 6186-6199, Jul 2020.

[16] A. Hassanien and S. A. Vorobyov, "Phased-MIMO Radar: A Tradeoff Between Phased-Array and MIMO Radars," IEEE Trans. Signal Process., vol. 58, no. 6, pp. 3137-3151, Feb 2010.

[17] F. Liu, C. Masouros, A. P. Petropulu, H. Griffiths, and L. Hanzo, "Joint Radar and Communication Design: Applications, State-of-the-Art, and the Road Ahead," IEEE Trans. Commun., vol. 68, no. 6, pp. 3834-3862, Jun 2020.

[18] A. M. Elbir and K. V. Mishra, "Joint antenna selection and hybrid beamformer design using unquantized and quantized deep learning networks," IEEE Trans. Wireless Commun., vol. 19, no. 3, pp. 16771688, March 2020.

[19] O. E. Ayach, S. Rajagopal, S. Abu-Surra, Z. Pi, and R. W. Heath, "Spatially sparse precoding in millimeter wave MIMO systems," IEEE Trans. Wireless Commun., vol. 13, no. 3, pp. 1499-1513, 2014.

[20] F. Liu and C. Masouros, "Hybrid Beamforming with Sub-arrayed MIMO Radar: Enabling Joint Sensing and Communication at mmWave Band," ICASSP 2019 - 2019 IEEE International Conference on Acoustics, Speech and Signal Processing (ICASSP), pp. 7770-7774, Dec 2017.

[21] Y. Xiao, F. Norouzian, E. G. Hoare, E. Marchetti, M. Gashinova, and M. Cherniakov, "Modeling and Experiment Verification of Transmissivity of Low-THz Radar Signal Through Vehicle Infrastructure," IEEE Sens. J., vol. 20, no. 15, pp. 8483-8496, Mar 2020. 